\title{
A Sub Class of K - Uniformly Starlike Functions with Negative Coefficients
}

\author{
R. B. Sharma , K.Saroja \\ Department of Mathematics Kakatiya University Warangal-506009 A.P., India \\ Department of Mathematics Govt. Degree College For Women Guntur-522022 A.P.,India.
}

Abstract: In this present paper we introduce a subclass of analytic functions with negative coefficients. We study coefficient bounds, distortion properties, covering theorem, extreme points, radius of close to convexity, star likeness, and convexity and integral transformations for the functions in this class. The results of this paper generalize many earlier results in this direction.

Mathematics subject classification: Primary 30C45

Keywords: Analytic functions, star like and convex functions, $k$-uniformly convex functions, negative coefficients.

\section{Introduction}

Let $A$ be the class of all analytic functions $f(z)=z+\sum_{n=2}^{\infty} a_{n} z^{n}$

defined on the open unit disc $U=\{z:|z|<1\}$

Let $S$ denote the subclass of $A$ consisting of functions that are univalent in $U$.

Let $S^{*}(\beta)$ and $C(\beta)$ be the classes of functions star like of order $\beta$ and convex of order $\beta(0 \leq \beta<1)$ respectively.

Let $\mathrm{T}$ be the subclass of $\mathrm{S}$, consisting of functions $f(z)=z-\sum_{n=2}^{\infty} a_{n} z^{n}, a_{n} \geq 0$

A function $f(z) \in T$ is called a function with negative coefficients and introduced by Silverman [7]. He investigated the star like and convex functions of order $\beta$ with negative coefficients. These classes are denoted by $S_{T} *(\beta)$ and $C_{T}(\beta)$ respectively.

Let $\operatorname{USF}(k, \beta)$ and $\operatorname{UCF}(k, \beta)$ be the classes consisting of the functions $f$ of the form (1.1) and satisfying the condition

$$
\begin{aligned}
& \operatorname{Re}\left\{\frac{z f^{\prime}(z)}{f(z)}-\beta\right\}>k\left|\frac{z f^{\prime}(z)}{f(z)}-1\right| \\
& \operatorname{Re}\left\{1+\frac{z f^{\prime \prime}(z)}{f^{\prime}(z)}-\beta\right\}>k\left|\frac{z f^{\prime \prime}(z)}{f^{\prime}(z)}\right|
\end{aligned}
$$

respectively for some $-1 \leq \beta<1, k \geq 0$ and $z \in U$.

These classes are known as k-uniformly star like functions of order $\beta$ and k-uniformly convex functions of order $\beta$ respectively. These classes are denoted by $\operatorname{USF}(\mathrm{k}), \mathrm{UCF}(\mathrm{k})$ respectively.

For $\beta=0$ in (1.3) and (1.4) we obtain the classes of k-uniformly star like and k-uniformly convex functions respectively. These classes have been extensively studied by Goodman [1, 2], Kanas and Srivastava [3], Kanas and Wisniowska [4], Ma and Minda [5] and Ronning [6].

In the present paper, we define a subclass of analytic functions and study the necessary and sufficient conditions, coefficient bounds, distortion properties, radii of star likeness, convexity and integral transformations for the functions in this class.

Definition 1.1 Let $\mathrm{R}(\lambda, k, \beta)$ be the class of functions $f \in A$ satisfying the condition

$\operatorname{Re}\left(\frac{z f^{\prime}(z)+\lambda z^{2} f^{\prime \prime}(z)}{f(z)}\right)>k\left|\frac{z f^{\prime}(z)+\lambda z^{2} f^{\prime \prime}(z)}{f(z)}-1\right|+\beta$ (1.5) 
for some $\lambda \geq 0, k \geq 0$ and $0 \leq \beta<1$. Here $R_{T}(\lambda, k, \beta)=R(\lambda, k, \beta) \cap T$

It is noted that

(i) $\quad R_{T}(0, k, 0)=U S F(k)$ defined and studied by S. Kanas and A. Wisniowska

(ii) $\quad R_{T}(0,0, \beta)=S_{T}^{*}(\beta)$ defined and studied by H. Silverman [7]

\section{A Characterization Theorem and resulting coefficient estimates}

2.1 We first find a sufficient condition for the functions $f(z) \in \mathcal{A}$ to be in the class $R(\lambda, k, \beta)$. We give a characterization of the class $R_{T}(\lambda, k, \beta)$ by finding a necessary and sufficient condition for function to be in $R_{T}(\lambda, k, \beta)$. This characterization also yields coefficient estimates for functions in this class.

Theorem 2.1: If $f \in \mathscr{A}$ satisfies the condition

$$
\sum_{n=2}^{\infty}[n(1+k)(n \lambda-\lambda+1)-(k+\beta)]\left|a_{n}\right| \leq(1-\beta)
$$

for some $\lambda \geq 0, k \geq 0$ and $0 \leq \beta<1$ then $f(z) \in R(\lambda, k, \beta)$.

Proof: Let $f \in \mathscr{A}$ and satisfies the condition (2.1)

To prove that $f(z) \in R(\lambda, k, \beta)$

Applying the principle

$$
\operatorname{Re}(w)>k|w-1|+\beta \Leftrightarrow \operatorname{Re}\left(w\left(1+k e^{i \theta}\right)-k e^{i \theta}\right)>\beta(-\pi \leq \theta \leq \pi, 0 \leq \beta<1, k \geq 0)
$$

for the function $w(z)=\frac{z f^{\prime}(z)+\lambda z^{2} f^{\prime \prime}(z)}{f(z)}$ on R.H.S we get

$$
\operatorname{Re}\left[\frac{\left[z f^{\prime}(z)+\lambda z^{2} f^{\prime \prime}(z)\right]\left[1+k e^{i \theta}\right]-k e^{i \theta} f(z)}{f(z)}\right]>\beta
$$

By setting $G(z)=\left[z f^{\prime}(z)+\lambda z^{2} f^{\prime \prime}(z)\right]\left[1+k e^{i \theta}\right]-k e^{i \theta} f(z)$ the above inequality (2.3) is equivalent to

$$
|G(z)+(1-\beta) f(z)|>|G(z)-(1+\beta) f(z)|
$$

Consider

$$
|G(z)+(1-\beta) f(z)|
$$

$=\left|\left[z f^{\prime}(z)+\lambda z^{2} f^{\prime \prime}(z)\right]\left[1+k e^{i \theta}\right]-k e^{i \theta} f(z)+(1-\beta) f(z)\right|$

Replacing $f(z), z f^{\prime}(z)$ and $z^{2} f^{\prime \prime}(z)$ with their equivalent series expansions in (2.4), we get

$$
\begin{aligned}
& |G(z)+(1-\beta) f(z)|= \\
& \quad\left|(2-\beta) z+\sum_{n=2}^{\infty}\left[[n(n \lambda-\lambda+1)+(1-\beta)]+k e^{i \theta}[n(n \lambda-\lambda+1)-1]\right] a_{n} z^{n}\right| \\
& \quad \geq(2-\beta)|z|-\sum_{n=2}^{\infty}[n(n \lambda-\lambda+1)+(1-\beta)]\left|a_{n}\right||z|^{n}-k \sum_{n=2}^{\infty}[n(n \lambda-\lambda+1)-1]\left|a_{n}\right||z|^{n}
\end{aligned}
$$

Similarly, we obtain

$$
\begin{aligned}
\mid G(z)- & (1+\beta) f(z) \mid \\
= & \left|\beta z-\sum_{n=2}^{\infty}\left[[n(n \lambda-\lambda+1)-(1+\beta)]+k e^{i \theta}[n(n \lambda-\lambda+1)-1]\right] a_{n} z^{n}\right| \\
& \leq \beta|z|+\sum_{n=2}^{\infty}[[n(n \lambda-\lambda+1)-(1+\beta)]+k[n(n \lambda-\lambda+1)-1]]\left|a_{n}\right||z|^{n}
\end{aligned}
$$

Therefore, from the inequalities (2.5) and (2.6) we have

$$
|G(z)+(1-\beta) f(z)|-|G(z)-(1+\beta) f(z)|
$$




$$
\begin{array}{r}
\geq 2(1-\beta)-\sum_{n=2}^{\infty} 2[n(1+k)(n \lambda-\lambda+1)-(k+\beta)]\left|a_{n}\right| \geq 0 \text { using the result in (2.1) } \\
\Rightarrow \operatorname{Re}\left(\frac{z f^{\prime}(z)+\lambda z^{2} f^{\prime \prime}(z)}{f(z)}\right)>k\left|\frac{\mid z f^{\prime}(z)+\lambda z^{2} f^{\prime \prime}(z)}{f(z)}-1\right|+\beta \text { from (2.2) }
\end{array}
$$

Hence $f(z) \in R(\lambda, k, \beta)$.

Theorem 2.2: $\quad$ A necessary and sufficient condition for a function $f \in T$ to be in the class

$R_{T}(\lambda, k, \beta)$ is that $\sum_{n=2}^{\infty}[n(1+k)(n \lambda-\lambda+1)-(k+\beta)] a_{n} \leq 1-\beta$ for some $k \geq 0, \lambda \geq 0$ and $0 \leq \beta<1$.

Proof: In view of theorem (2.1), it is sufficient to show that if $f \in R_{T}(\lambda, k, \beta)$ then $f$ satisfies the condition (2.1)

Suppose that $f(z)=z-\sum_{n=2}^{\infty} a_{n} z^{n}$ is in $R_{T}(\lambda, k, \beta)$

By setting $0 \leq|z|=r<1$ and choosing the values of $\mathrm{z}$ on the real axis, then from the inequality (2.3), we have

$$
\operatorname{Re}\left(\frac{r-\sum_{n=2}^{\infty}\left[n+n \lambda(n-1)+k e^{i \theta}[n+n \lambda(n-1)-1]\right] a_{n} r^{n}}{r-\sum_{n=2}^{\infty} a_{n} r^{n}}\right)>\beta
$$

Since $\operatorname{Re}\left|-e^{i \theta}\right| \geq-\left|e^{i \theta}\right|=-1$.

The above inequality (2.7) reduces to

$$
\operatorname{Re}\left(\frac{(1-\beta)-\sum_{n=2}^{\infty}[n(1+k)(n \lambda-\lambda+1)-(k+\beta)] a_{n} r^{n-1}}{1-\sum_{n=2}^{\infty} a_{n} r^{n-1}}\right) \geq 0
$$

Letting $r \rightarrow 1$ in (2.8) we get

$$
\sum_{n=2}^{\infty}[n(1+k)(n \lambda-\lambda+1)-(k+\beta)] a_{n} \leq(1-\beta)
$$

This is the result in (2.1)

Hence the theorem.

Corollary 2.2: If $f(z)$ is in $R_{T}(\lambda, k, \beta)$ then

$$
a_{n} \leq \frac{1-\beta}{[n(1+k)(n \lambda-\lambda+1)-(k+\beta)]} \quad \forall n \geq 2
$$

This result is sharp for each $\mathrm{n}$ for functions of the form

$$
f_{n}(z)=z-\frac{1-\beta}{[n(1+k)(n \lambda-\lambda+1)-(k+\beta)]} z^{n} \quad \forall n \geq 2
$$

\section{Distortion and covering theorems for the function class $R_{T}(\lambda, k, \beta)$}

Theorem 3.1: If the function $f \in R_{T}(\lambda, k, \beta)$ then

$$
r-\frac{1-\beta}{[2(1+k)(1+\lambda)-(k+\beta)]} r^{2} \leq|f(z)| \leq r+\frac{1-\beta}{[2(1+k)(1+\lambda)-(k+\beta)]} r^{2}, \quad \forall 0<|z|=r<1
$$


The equality in (3.1) is attained for the function $f$ given by

$$
f(z)=z-\frac{1-\beta}{[2(1+k)(1+\lambda)-(k+\beta)]} z^{2}
$$

Proof: Since $f(z) \in R_{T}(\lambda, k, \beta)$, from the inequality (2.1) we have

$$
\sum_{n=2}^{\infty}[n(1+k)(n \lambda-\lambda+1)-(k+\beta)] a_{n} \leq 1-\beta
$$

It is easily known that

$$
\begin{aligned}
& {[2(1+k)(1+\lambda)-(k+\beta)] \sum_{n=2}^{\infty} a_{n} \leq \sum_{n=2}^{\infty}[n(1+k)(n \lambda-\lambda+1)-(k+\beta)] a_{n} \leq 1-\beta} \\
& \Rightarrow \sum_{n=2}^{\infty} a_{n} \leq \frac{1-\beta}{[2(1+k)(1+\lambda)-(k+\beta)]} \quad|f(z)|=\left|z-\sum_{n=2}^{\infty} a_{n} z^{n}\right| \leq|z|+\sum_{n=2}^{\infty} a_{n}|z|^{n} \\
& \text { Consider } \quad \leq r+\frac{1-\beta}{[2(1+k)(1+\lambda)-(k+\beta)]} r^{2} \text { [Using the inequality 3.3] }
\end{aligned}
$$

This gives the right hand side of inequality (3.1).

$$
\text { Also } \begin{aligned}
|f(z)| & =\left|z-\sum_{n=2}^{\infty} a_{n} z^{n}\right| \geq|z|-\sum_{n=2}^{\infty} a_{n}|z|^{n} \\
& \geq r-r^{2} \sum_{n=2}^{\infty} a_{n} \geq r-\frac{1-\beta}{[2(1+k)(1+\lambda)-(k+\beta)]} r^{2}
\end{aligned}
$$

which is the left hand side of (3.1).

It can be easily seen that the function $f(z)$ defined by (3.2) is the extremal for the theorem.

Theorem 3.2: If $f \in R_{T}(\lambda, k, \beta)$, then

$$
1-\frac{2(1-\beta)}{[2(1+k)(1+\lambda)-(k+\beta)]} r \leq\left|f^{\prime}(z)\right| \leq 1+\frac{2(1-\beta)}{[2(1+k)(1+\lambda)-(k+\beta)]} r \quad \forall 0<|z|=r<1
$$

The equality in (3.4) holds true for the function $f$ given by (3.2).

Proof: Since $f \in R_{T}(\lambda, k, \beta)$ we have

$$
\begin{array}{r}
\left|f^{\prime}(z)\right| \leq 1+\sum_{n=2}^{\infty} n a_{n}|z|^{n-1} \leq 1+r \sum_{n=2}^{\infty} n a_{n} \\
\text { and }\left|f^{\prime}(z)\right| \geq 1-\sum_{n=2}^{\infty} n a_{n}|z|^{n-1} \geq 1-r \sum_{n=2}^{\infty} n a_{n}
\end{array}
$$

The result in (3.4) holds true from (3.5), (3.6) and using the simple consequence of (3.3) given by

$$
\sum_{n=2}^{\infty} n a_{n} \leq \frac{2(1-\beta)}{[2(1+k)(1+\lambda)-(k+\beta)]}
$$

The result is sharp for the function $f$ given in (3.2).

\section{Closure Theorems for the class $R_{T}(\lambda, k, \beta)$}

In the next theorems we prove that the class $R_{T}(\lambda, k, \beta)$ is closed under convex linear combinations.

$$
\text { Theorem 4.1: If } f_{1}(z)=z \text { and } f_{n}(z)=z-\frac{(1-\beta) z^{n}}{[n(1+k)(n \lambda-\lambda+1)-(k+\beta)]}, n \geq 2
$$


Then $f \in R_{T}(\lambda, k, \beta)$ if and only if it can be expressed in the form $f(z)=\sum_{n=1}^{\infty} \lambda_{n} f_{n}(z)$ where $\lambda_{n} \geq 0$ and $\sum_{n=1}^{\infty} \lambda_{n}=1$

Proof: Suppose $f(z)=\sum_{n=1}^{\infty} \lambda_{n} f_{n}(z)$ with $\lambda_{n} \geq 0$ and $\sum_{n=1}^{\infty} \lambda_{n}=1$.

Since $\sum_{n=1}^{\infty} \lambda_{n} f_{n}(z)=\lambda_{1} f_{1}(z)+\sum_{n=2}^{\infty} \lambda_{n} f_{n}(z)$

$$
\begin{aligned}
& =\left[1-\sum_{n=2}^{\infty} \lambda_{n}\right] f_{1}(z)+\sum_{n=2}^{\infty} \lambda_{n}\left[z-\frac{(1-\beta)}{[n(1+k)(n \lambda-\lambda+1)-(k+\beta)]} z^{n}\right] \\
& =\left[1-\sum_{n=2}^{\infty} \lambda_{n}\right] z+\sum_{n=2}^{\infty} \lambda_{n}\left[z-\frac{(1-\beta)}{[n(1+k)(n \lambda-\lambda+1)-(k+\beta)]} z^{n}\right] \\
& =z-\sum_{n=2}^{\infty} \lambda_{n} \frac{(1-\beta)}{[n(1+k)(n \lambda-\lambda+1)-(k+\beta)]} z^{n}
\end{aligned}
$$

Consider $\sum_{n=2}^{\infty} \lambda_{n} \frac{(1-\beta)}{[n(1+k)(n \lambda-\lambda+1)-(k+\beta)]} \cdot \frac{[n(1+k)(n \lambda-\lambda+1)-(k+\beta)]}{(1-\beta)}$

$$
=\sum_{n=2}^{\infty} \lambda_{n}=1-\lambda_{1} \leq 1
$$

Thus the coefficients of $f(z)$ satisfy the inequality (2.1).

Hence from the Theorem (2.2) it follows that $f \in R_{T}(\lambda, k, \beta)$.

Conversely suppose $f \in R_{T}(\lambda, k, \beta)$

Since $a_{n} \leq \frac{(1-\beta)}{[n(1+k)(n \lambda-\lambda+1)-(k+\beta)]}, \quad n \geq 2$

Setting $\lambda_{n}=\frac{[n(1+k)(n \lambda-\lambda+1)-(k+\beta)]}{(1-\beta)} a_{n}, \quad n=2,3 \ldots$

and $\lambda_{1}=1-\sum_{n=2}^{\infty} \lambda_{n}$ then $f(z)=\sum_{n=1}^{\infty} \lambda_{n} f_{n}(z)$

This completes the proof of the theorem.

Theorem 4.2: The class $R_{T}(\lambda, k, \beta)$ is closed under convex linear combinations.

Proof: Suppose that each of the function $f_{l}(z)$ given by

$$
f_{l}(z)=z-\sum_{n=2}^{\infty} a_{n, l} z^{n}, \quad(l=1,2) \text { is in the class } R_{T}(\lambda, k, \beta) .
$$

We need to prove that the function $H(z)$ given by $H(z)=\lambda_{1} f_{1}(z)+\left(1-\lambda_{1}\right) f_{2}(z)\left(0 \leq \lambda_{1} \leq 1\right)$ also lies in the class $R_{T}(\lambda, k, \beta)$.

$$
\begin{aligned}
& \text { Since } H(z)=z-\sum_{n=2}^{\infty}\left[\lambda_{1} a_{n, 1}+\left(1-\lambda_{1}\right) a_{n, 2}\right] \cdot z^{n} \\
& \text { Consider } \sum_{n=2}^{\infty}[n(1+k)(n \lambda-\lambda+1)-(k+\beta)]\left[\lambda_{1} a_{n, 1}+\left(1-\lambda_{1}\right) a_{n, 2}\right] \\
& =\lambda_{1} \sum_{n=2}^{\infty}[n(1+k)(n \lambda-\lambda+1)-(k+\beta)] a_{n, 1}+\left(1-\lambda_{1}\right) \sum_{n=2}^{\infty}[n(1+k)(n \lambda-\lambda+1)-(k+\beta)] a_{n, 2} \\
& \leq \lambda_{1}(1-\beta)+\left(1-\lambda_{1}\right)(1-\beta) \leq 1-\beta
\end{aligned}
$$


Thus from the theorem (2.2), $H(z) \in R_{T}(\lambda, k, \beta)$. Hence the class $R_{T}(\lambda, k, \beta)$ is closed under convex linear combinations.

\section{Radii of star likeness, convexity and close to convexity for the functions in the class $\boldsymbol{R}_{T}(\lambda, \boldsymbol{k}, \boldsymbol{\beta})$}

In the next theorems we determine radius of star likeness, convexity and close to convexity for the functions in the class $R_{T}(\lambda, k, \beta)$

Theorem 5.1: If $f \in R_{T}(\lambda, k, \beta)$ then $f$ is star like of order $\rho(0 \leq \rho<1)$ in $|z|<\gamma_{1}(\lambda, k, \beta, \rho)$ where $\gamma_{1}(\lambda, k, \beta, \rho)=\inf _{n}\left[\frac{(1-\rho)[n(1+k)(n \lambda-\lambda+1)-(k+\beta)]}{(n-\rho)(1-\beta)}\right]^{1 / n-1} \forall n \geq 2$ and the result is sharp.

Proof: Suppose $f \in R_{T}(\lambda, k, \beta)$. It is sufficient to show that

$$
\left|\frac{z f^{\prime}(z)}{f(z)}-1\right| \leq 1-\rho \quad\left[\text { for } 0 \leq \rho<1,|z|<\gamma_{1}(\lambda, k, \beta, \rho)\right]
$$

Replacing $f(z), z f^{\prime}(z)$ in the left hand side of (5.1) with their equivalent expressions in series, we get

$$
\left|\frac{\sum_{n=2}^{\infty}(n-1) a_{n} z^{n-1}}{1-\sum_{n=2}^{\infty} a_{n} z^{n-1}}\right| \leq \frac{\sum_{n=2}^{\infty}(n-1) a_{n}|z|^{n-1}}{1-\sum_{n=2}^{\infty} a_{n}|z|^{n-1}}
$$

This will be bounded by $(1-\rho)$ if

$$
\begin{aligned}
& \sum_{n=2}^{\infty}(n-1) a_{n}|z|^{n-1} \leq(1-\rho)\left[1-\sum_{n=2}^{\infty} a_{n}|z|^{n-1}\right] \\
& \sum_{n=2}^{\infty} \frac{(n-\rho)}{(1-\rho)}|z|^{n-1} a_{n} \leq 1 \ldots . . \text { (5.2) }
\end{aligned}
$$

Since for $f \in R_{T}(\lambda, k, \beta)$, from Theorem (2.2) we have

$$
\sum_{n=2}^{\infty}\left[\frac{n(1+k)(n \lambda-\lambda+1)-(k+\beta)}{1-\beta}\right] a_{n} \leq 1
$$

The condition (5.2) will be satisfied if

$$
\begin{aligned}
& \frac{n-\rho}{1-\rho}|z|^{n-1} \leq\left[\frac{n(1+k)(n \lambda-\lambda+1)-(k+\beta)}{1-\beta}\right] \quad \text { for each } n \geq 2 . \\
& \Rightarrow|z| \leq\left[\frac{(1-\rho)[n(1+k)(n \lambda-\lambda+1)-(k+\beta)]}{(n-\rho)(1-\beta)}\right]^{1 / n-1}
\end{aligned}
$$

Setting $|z|=r_{1}(\lambda, k, \beta, \rho)$, the result of the theorem follows. And the result is sharp for each $n$ for the functions $f_{n}(z)$ given in (2.9)

The proof of the theorem is thus complete.

Theorem 5.2: If $f \in R_{T}(\lambda, k, \beta)$ then $f$ is convex of order $\rho(0 \leq \rho<1)$ in $|z|<\gamma_{2}(\lambda, k, \beta, \rho)$ where $\gamma_{2}(\lambda, k, \beta, \rho)=\inf _{n}\left[\frac{(1-\rho)[n(1+k)(n \lambda-\lambda+1)-(k+\beta)]}{n(n-\rho)(1-\beta)}\right]^{1 / n-1} n \geq 2 \quad$ and the result is sharp. 
Proof: Suppose $f \in R_{T}(\lambda, k, \beta)$. It is sufficient to show that

$$
\left|\frac{z f^{\prime \prime}(z)}{f^{\prime}(z)}\right| \leq 1-\rho\left[\text { for } 0 \leq \rho<1,|z|<\gamma_{2}(\lambda, k, \beta, \rho)\right]
$$

Replacing $f^{\prime}(z)$ and $z f^{\prime \prime}(z)$ in the left hand side of (5.3) with their equivalent expressions in series, then we get

$$
\left|\frac{\sum_{n=2}^{\infty} n(n-1) a_{n} z^{n-1}}{1-\sum_{n=2}^{\infty} n a_{n} z^{n-1}}\right| \leq \frac{\sum_{n=2}^{\infty} n(n-1) a_{n}|z|^{n-1}}{1-\sum_{n=2}^{\infty} n a_{n}|z|^{n-1}}
$$

This will be bounded by $(1-\rho)$ if

$$
\begin{aligned}
& \sum_{n=2}^{\infty} n(n-1) a_{n}|z|^{n-1} \leq(1-\rho)\left[1-\sum_{n=2}^{\infty} n a_{n}|z|^{n-1}\right] \\
& \text { or } \quad \sum_{n=2}^{\infty} \frac{n(n-\rho)}{(1-\rho)} a_{n}|z|^{n-1} \leq 1 \quad \ldots . . \text { (5.4) }
\end{aligned}
$$

For $f \in R_{T}(\lambda, k, \beta)$, from the Theorem (2.2) we have

$$
\sum_{n=2}^{\infty}\left[\frac{n(1+k)(n \lambda-\lambda+1)-(k+\beta)}{1-\beta}\right] a_{n} \leq 1
$$

The condition (5.4) will be satisfied if

$$
\begin{aligned}
& \frac{n(n-\rho)}{(1-\rho)}|z|^{n-1} \leq\left[\frac{n(1+k)(n \lambda-\lambda+1)-(k+\beta)}{1-\beta}\right] \text { for each } n \geq 2 . \\
& \Rightarrow|z| \leq\left[\frac{(1-\rho)[n(1+k)(n \lambda-\lambda+1)-(k+\beta)]}{n(n-\rho)(1-\beta)}\right]^{1 / n-1} n=2,3 \ldots .
\end{aligned}
$$

Setting $|z|=\gamma_{2}(\lambda, k, \beta, \rho)$, then the result of the theorem follows.

Sharpness of this result can be easily verified for functions $f_{n}(z)$ stated as in (2.9)

Theorem 5.3: If $f \in R_{T}(\lambda, k, \beta)$ then $f$ is close to convex of order $\rho(0 \leq \rho<1)$ in $|z|<\gamma_{3}(\lambda, k, \beta, \rho)$ where

$$
\gamma_{3}(\lambda, k, \beta, \rho)=\inf _{n}\left[\frac{(1-\rho)[n(1+k)(n \lambda-\lambda+1)-(k+\beta)]}{n(1-\beta)}\right]^{1 / n-1}, \forall n \geq 2 \text { and the result is sharp. }
$$

Proof: Suppose $f \in R_{T}(\lambda, k, \beta)$. It is sufficient to show that

$$
\left|f^{\prime}(z)-1\right| \leq 1-\rho\left[\text { for } 0 \leq \rho<1,|z|<\gamma_{3}(\lambda, k, \beta, \rho)\right]
$$

Replacing $f^{\prime}(z)$ in the left hand side of (5.5) with its equivalent expression in series, then we get

$\left|1-\sum_{n=2}^{\infty} n a_{n} z^{n-1}-1\right| \leq \sum_{n=2}^{\infty} n a_{n}|z|^{n-1}$

This will be bounded by $(1-\rho)$ if

$$
\begin{array}{r}
\sum_{n=2}^{\infty} n a_{n}|z|^{n-1} \leq 1-\rho \\
\sum_{n=2}^{\infty}\left(\frac{n}{1-\rho}\right) a_{n}|z|^{n-1} \leq 1
\end{array}
$$


Since for $f \in R_{T}(\lambda, k, \beta)$, from the Theorem (2.2) we have

$$
\begin{aligned}
& \sum_{n=2}^{\infty}\left[\frac{n(1+k)(n \lambda-\lambda+1)-(k+\beta)}{1-\beta}\right] a_{n} \leq 1 \\
& \text { The condition (5.6) will be satisfied if } \\
& \left(\frac{n}{1-\rho}\right)|z|^{n-1} \leq\left[\frac{n(1+k)(n \lambda-\lambda+1)-(k+\beta)}{1-\beta}\right] \text { for each } n \geq 2 . \\
|z| \leq & {\left[\frac{(1-\rho)[n(1+k)(n \lambda-\lambda+1)-(k+\beta)]}{n(1-\beta)}\right]^{1 / n-1} \forall n \geq 2 } \\
& \text { Setting }|z|=\gamma_{3}(\lambda, k, \beta, \rho), \text { the result follows. }
\end{aligned}
$$

Sharpness of this result can be easily verified for functions $f_{n}(z)$ as stated in (2.9)

Theorem 5.4: If $f(z) \in R_{T}(\lambda, k, \beta)$ then $f(z) \in T^{*}(\delta)$ where

$\delta(\lambda, k, \beta)=1-\frac{(1-\beta)}{[2(1+k)(1+\lambda)-(k+\beta)]-(1-\beta)}$ and the result is sharp.

Proof: Suppose $f \in R_{T}(\lambda, k, \beta)$. It is sufficient to show that

$\sum_{n=2}^{\infty}\left(\frac{n-\delta}{1-\delta}\right) a_{n} \leq 1$

Since for $f \in R_{T}(\lambda, k, \beta)$, from the Theorem (2.2) we have

$\sum_{n=2}^{\infty}\left[\frac{n(1+k)(n \lambda-\lambda+1)-(k+\beta)}{1-\beta}\right] a_{n} \leq 1$

Thus (5.7) will be satisfied if

$$
\begin{aligned}
\left(\frac{n-\delta}{1-\delta}\right) \leq \frac{[n(1+k)(n \lambda-\lambda+1)-(k+\beta)]}{1-\beta} \text { for each } n \\
\delta \leq \frac{[n(1+k)(n \lambda-\lambda+1)-(k+\beta)]-n(1-\beta)}{[n(1+k)(n \lambda-\lambda+1)-(k+\beta)]-(1-\beta)} \forall n \geq 2 \\
\delta \leq 1-\frac{(n-1)(1-\beta)}{[n(1+k)(n \lambda-\lambda+1)-(k+\beta)]-(1-\beta)} \ldots . . \\
=\psi(n)
\end{aligned}
$$

Since for $\psi(n) \geq \psi(2) \quad \forall n \geq 2, \lambda \geq 0, k \geq 0$ and, $0 \leq \beta<1$ putting $n=2$ in (5.8) we get

$$
\delta \leq 1-\frac{(1-\beta)}{[2(1+k)(1+\lambda)-(k+\beta)]-(1-\beta)}
$$

Hence the result

The sharpness of this result can be easily verified with the extremal function given in (3.2). This completes the proof of the theorem.

\section{Integral Operators}

In the next theorem we consider the integral operators of functions in the class $R_{T}(\lambda, k, \beta)$

Theorem 6.1: If $f \in R_{T}(\lambda, k, \beta)$ then the function $F(z)$ defined by

$$
F(z)=\frac{1+c}{z^{c}} \int_{0}^{c} t^{c-1} f(t) d t \quad(c>-1) \text { is also in } R_{T}(\lambda, k, \beta)
$$

Proof: Suppose $f \in R_{T}(\lambda, k, \beta)$, from (6.1) we have 


$$
F(z)=z-\sum_{n=2}^{\infty}\left(\frac{c+1}{c+n}\right) a_{n} z^{n} \quad 0<\frac{c+1}{c+n}<1
$$

Consider $\sum_{n=2}^{\infty}[n(1+k)(n \lambda-\lambda+1)-(k+\beta)] a_{n}\left(\frac{c+1}{c+n}\right)$

$\leq \sum_{n=2}^{\infty}[n(1+k)(n \lambda-\lambda+1)-(k+\beta)] a_{n}$

$\leq 1-\beta$ (Using the inequality $(2.1)$

$\Rightarrow F(z) \in R_{T}(\lambda, k, \beta)$

Hence the theorem

\section{Acknowledgements.}

The authors are thankful to Prof.T.Ramreddy for his valuable guidance in making this paper and the referee for the suggestions.

\section{References:}

[1] Goodman A.W.:- "On uniformly convex functions", Ann. Pal. Math. 56 (1991), 87-92.

[2] Goodman A.W.: "On uniformly starlike functions" J.of Math. Anal And Appl. 155 (1991), 364-370.

[3] Kanas.S and Srivastava.H.M.: Linear operators associated with k-uniformly convex functions, Integral transforms Spec. Funct., 9, (2000), $121-132$.

[4] Wisniowska. A. and Kanas. S: Conic regions and K-uniform convexity, J. Comput, Appl. Math., 105 (1999), $327-336$.

[5] Ma.W and Minda.D: Uniformly convex functions, Ann, Polon, Math, 57 (1992), 165 - 175.

[6] Ronning. F.:- "Uniformly convex functions and a corresponding class of starlike functions, proc. Amer. Math. Soc. 118 (1993), 189 196.

[7] Silverman H.:- Univalent functions with negative coefficients, Proc. Amer. Math. Soc. 51(1975), 109-116. 